Europhysics Letters

PREPRINT

\title{
Bubble kinetics in a steady-state column of aqueous foam
}

\author{
K. Feitosa, Olivia L. Halt, Randall D. Kamien, and D. J. Durian \\ Department of Physics and Astronomy, University of Pennsylvania, 209 S. 33rd Street, \\ Philadelphia, PA 19104-6396, USA
}

PACS. 83.80.Iz - Emulsions and foams.

PACS. 82.70.Rr - Aerosols and foams.

\begin{abstract}
We measure the liquid content, the bubble speeds, and the distribution of bubble sizes, in a vertical column of aqueous foam maintained in steady-state by continuous bubbling of gas into a surfactant solution. Nearly round bubbles accumulate at the solution/foam interface, and subsequently rise with constant speed. Upon moving up the column, they become larger due to gas diffusion and more polyhedral due to drainage. The size distribution is monodisperse near the bottom and polydisperse near the top, but there is an unexpected range of intermediate heights where it is bidisperse with small bubbles decorating the junctions between larger bubbles. We explain the evolution in both bidisperse and polydisperse regimes, using Laplace pressure differences and taking the liquid fraction profile as a given.
\end{abstract}

Aqueous foam is a quintessential non-equilibrium system, even in absence of film rupture. An initially homogeneous foam will drain due to gravity, and will coarsen due to gas diffusion, en route to an equilibrium state of total phase separation where the foam vanishes. The beautiful topology and microstructure of soap films and their junctions into Plateau borders and vertices have inspired wide-ranging studies of coarsening and drainage as fundamental evolution mechanisms [1,2]. However, drier foams coarsen more rapidly, and coarser foams drain more rapidly, and this interplay enhances temporal evolution and spatial inhomogeneity in situations such as free [3] and forced drainage [4]. In spite of good progress, several open questions remain. For example there is no consensus on how the coarsening rate depends on liquid content [4-6]. And there is little understanding of how drainage is affected by a distribution of bubble sizes.

To advance the understanding of such issues we examine a geometry in which a vertical column of foam is created by a continuous stream of small bubbles into a pool of surfactant solution. Here the foam reaches a state where the bubbles rise at constant speed, and the liquid remains at rest, in the laboratory frame. Prior studies of such steady-state foams focus mainly on the height of the foam as the key observable quantity $[7,8]$, though recently the liquid fraction profile was predicted under the assumption of constant bubble size [9]. Here the liquid content, the bubble speed, and the bubble size distribution, are all independent of time and are hence measured at our leisure as a function of height. This is a simplification over free and forced drainage, which require study as a function of both position and time. Furthermore, the steady-state condition allows for a different and unexplored type of interplay

(C) EDP Sciences 
between drainage and coarsening. As presented below, our measurements reveal two striking qualitative features that can be quantitatively modeled: the formation of a bidisperse bubble size distribution, and a rate of coarsening that increases without apparent bound for decreasing liquid fractions.

Methods and data. - We generate a steady-state column of foam by continuously blowing gas into a surfactant solution inside a tall Lucite cylinder, $61 \mathrm{~cm}$ in height and $5.08 \mathrm{~cm}$ in inner diameter. The bottom of the cylinder is filled with $120 \mathrm{ml}$ of an aqueous solution of AOS ( $\alpha$ olefin sulfonate, Bio-Terge AS-40 CG-P, Stepan Company) and $\mathrm{NaCl}$ with concentrations of $0.4 \%$ and $0.01 \%$ by weight respectively. The solution has surface tension $\gamma=44 \mathrm{dyne} / \mathrm{cm}$ and viscosity $\mu=0.011 \mathrm{~g} / \mathrm{cm}$-s. Small bubbles of $\mathrm{CO}_{2}$ (Airgas East, $99.2 \%$ pure, $\mathrm{H}_{2} \mathrm{O}<200 \mathrm{ppm}$, $\left.\mathrm{NH}_{3}<25 \mathrm{ppm}, \mathrm{CO}<10 \mathrm{ppm}, \mathrm{H}_{2} \mathrm{~S} / \mathrm{SO}_{2}<5 \mathrm{ppm}, \mathrm{NO}_{X}<2.5 \mathrm{ppm}\right)$ are sparged into the solution through a fine mesh cloth. This gas has solubility $1.17 \mathrm{~mol} / \mathrm{l}$ and diffusivity $1.8 \times$ $10^{5} \mathrm{~cm}^{2} / \mathrm{s}$ in water. The flow rate of $\mathrm{CO}_{2}$ is held fixed and is measured by an electronic flow meter (Omega, model FMA 3102) to be $0.505 \mathrm{ml} / \mathrm{s}$; the resulting gas flux is $0.029 \pm 0.001 \mathrm{~cm} / \mathrm{s}$, where the error is dominated by calibration uncertainty. After six hours, a steady state is achieved in which the foam is about $45 \mathrm{~cm}$ high and where the input of small bubbles at the bottom is balanced by the bursting of large bubbles into the atmosphere at the top. Visually, there is no bursting anywhere in the foam except at the top free surface.

The physical properties of the steady-state foam are measured as follows. First, the liquid volume fraction $\varepsilon(z)$ is deduced from electrical conductivity vs height $z$. A series of 19 stainless steel electrodes, $1.27 \mathrm{~cm}$ in diameter and $2.54 \mathrm{~cm}$ apart, are attached to two acetal strips placed opposite one another inside the foam column. The conductivities of liquid and foam are both measured by an impedance meter (1715 LCR Digibridge, QuadTech) configured to measure the resistance of a parallel resistor-capacitor equivalent circuit, operated at a frequency of $1 \mathrm{kHz}$ and voltage level of $1.00 \mathrm{~V}$. At this frequency, the capacitive contribution of the foam is negligible. The conductivity is measured at smaller intervals than $2.54 \mathrm{~cm}$, both by connecting diagonally-opposite electrodes and by changing the height of the pool of surfactant solution relative to the electrode heights. The liquid fraction is calculated from the ratio of foam to liquid conductivity, $\sigma=\sigma_{\text {foam }} / \sigma_{\text {liquid }}$, using the semi-empirical relation $\varepsilon=3 \sigma(1+11 \sigma) /\left(1+25 \sigma+10 \sigma^{2}\right)[10]$. Results for $\varepsilon(z)$ are displayed on the left-hand axis in Fig. 1 for several different ages of the sample. Indeed the data are independent of time, indicative of a steady-state. The foam is wetter on the bottom and drier towards the top. At the very top, where the bubbles burst, surfactant gradually accumulates and causes an uncontrolled increase in liquid conductivity; this gives an erroneous apparent increase in liquid fraction with time.

Next, the upward speed $u(z)$ of the bubbles at a given height is deduced from a rapid sequence of digital photographs (Nikon D70; AF Micro Nikkor lens, 60 mm, 1:2.8D). Example images are given in Fig. [2 The average bubble displacement is calculated as the location of the peak of the vertical cross-correlation of two subsequent images. The time between images is $0.333 \mathrm{~s}$, chosen so that the bubbles move noticeably without significant change in the field of view or the packing structure. Values for ten successive pairs of images are averaged together. Final bubble speed data are displayed vs height on the right-axis of Fig 1 The speeds are constant, $u=0.032 \pm 0.003 \mathrm{~cm} / \mathrm{s}$, independent of height. This value is consistent with the directly-measured gas flux, $0.029 \pm 0.001 \mathrm{~cm} / \mathrm{s}$. Furthermore, we observe no global convection or swirling. Therefore, the bubble motion is a constant plug-flow across the column.

Finally, image analysis is performed to identify individual bubbles and the area $A$ they occupy on the column surface. The equivalent radius is taken as $R=\sqrt{A / \pi}$; results are not adjusted according to Ref. [11], which assumes that the surface bubbles are equivalent to a 


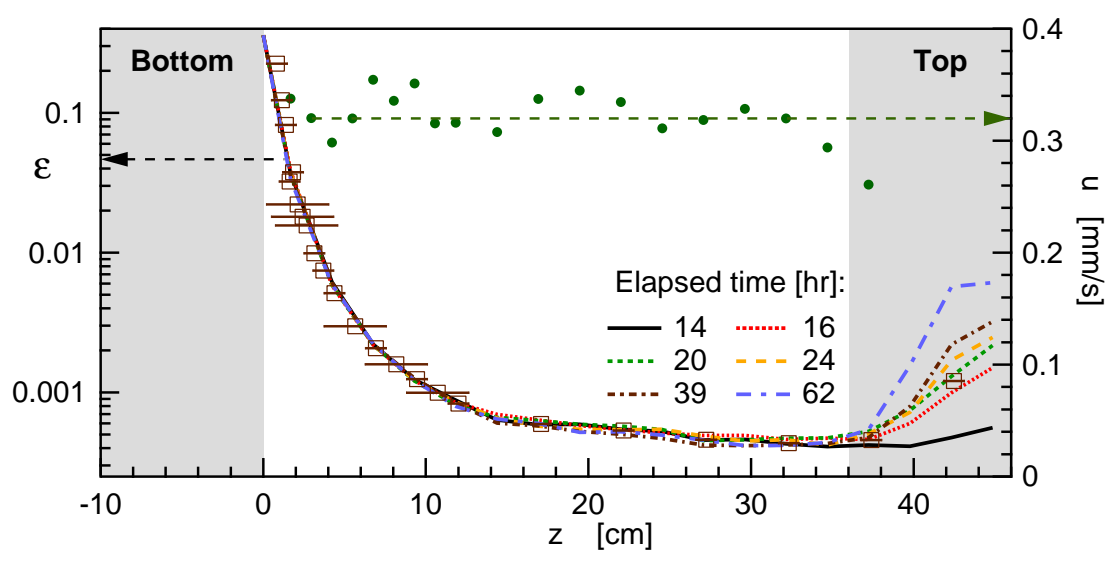

Fig. 1 - Liquid fraction $\varepsilon$ (left) and average bubble speed $u$ (right) as a function of height. The liquid fraction data are for two runs; the first is vs time (curves), the second is for varied electrode heights (squares); horizontal error bars indicate the range over which conductivity was measured. The bottom gray area represents the surfactant solution; the top gray area represents the region where bubbles burst.

random cut in a bulk foam. For heights greater than $z=1 \mathrm{~cm}$ (which includes both bidisperse and polydisperse regimes - see below), we are able to inspect the region 2-3 bubbles from the surface; we notice no qualitative difference from boundary bubbles. A scatter plot of radius vs height is displayed in Fig. 3 with one small point for each measured bubble. For heights $0<z<0.7 \mathrm{~cm}$ near the bottom of the column the bubbles are small and the distribution is relatively monodisperse; the average and standard deviation are $\langle R\rangle=0.0154 \mathrm{~cm}$ and $\sigma_{o}=0.0022 \mathrm{~cm}$. For increasing heights $z>2.5 \mathrm{~cm}$ through most of the column, the bubbles become progressively larger and the size distribution broadens. The breadth of the distribution can be gauged by the dimensionless variance $v=\left\langle R^{2}\right\rangle /\langle R\rangle^{2}-1$, and by the polydispersity

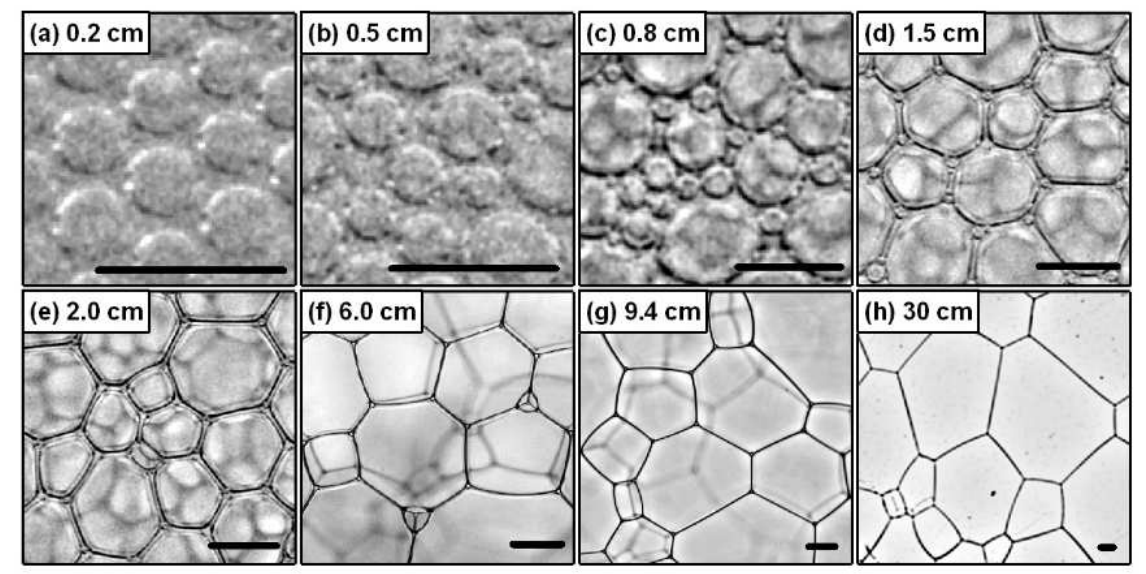

Fig. 2 - Example surface images at different heights, as labeled; scale bars are $1 \mathrm{~mm}$. These images were acquired after steady-state was established. 
parameter $p=\left\langle R^{3}\right\rangle^{2 / 3} /\left\langle R^{2}\right\rangle-1[19]$. These quantities are shown vs height in the bottom plot of Fig. [3] Both start small, develop a transient overshoot, and become constant at large heights, $v=0.27 \pm 0.11$ and $p=0.18 \pm 0.06$. For $z>3 \mathrm{~cm}$ we therefore scale the individual radii by a running average, and combine all the data into a single dimensionless size distribution. The result (Fig. 3 inset) is similar to Fig. 18 of Ref. [12], and is well-fit by a Weibull form.

For a narrow range of intermediate heights, roughly $0.7 \mathrm{~cm}<z<2.5 \mathrm{~cm}$, the bubble radii data in Fig. 3 fall into two distinct branches. The size distribution doesn't smoothly broaden as a function of height in going from monodisperse to polydisperse regions; rather, it develops a bidisperse region and becomes polydisperse only after the branch of small bubbles disappears. Note that the existence of a bidisperse region can be seen directly by inspection of surface images. At the bottom, Figs. 2 a-b, the bubbles are monodisperse; towards the top, Figs. 2]-h, the bubbles are polydisperse with larger and smaller bubbles arrayed at random; in between, Figs. 25-e, the junctions between big bubbles are all decorated with small bubbles. The small "decorating" bubbles become progressively smaller in Figs. 2 $\mathrm{d}$-e, and may best be viewed by enlarging the figure on-screen.

This surprising observation has no precedent, to our knowledge. Prior experiments on the evolution of initially-monodisperse bulk foams all found smooth relaxation to a polydisperse size distribution with no intermediate bidisperse regime $[4,13,14]$. Perhaps the closest connection is with Fig. 2 of Ref. [15], where coarsening was depicted for an initially-ordered two-dimensional foam. As here, that sample developed two distinct populations and became uniformly polydisperse only after the population of small bubbles fully disappeared. Furthermore the second moment $\mu_{2}$ of the side distribution did not increase monotonically from a small value, but rather developed a transient overshoot at intermediate times [16], much like the polydispersity parameter here. However, by contrast with our sample, the small bubbles and big bubbles were separately clustered together in segregated domains.

Models. - We now attempt to model the observed behavior of bubble size vs height in the column, with the measured liquid-fraction profile as a given. Since the vertical bubble speed is a constant equal to the gas flux per unit area, $u=0.032 \mathrm{~cm} / \mathrm{s}$, and since there is no convection or film rupture, the basic physics is controlled solely by the diffusion of gas from smaller to larger bubbles. Ultimately this is driven by surface tension, where the smaller bubbles are under higher Laplace pressure, and is a means of reducing total interfacial area. Furthermore, time- and height- derivatives are related by $\mathrm{d} / \mathrm{d} t=u \mathrm{~d} / \mathrm{d} z$. The approach developed by Lemlich [17] and by Markworth [18] is similar to earlier work on the kinetics of phase separation in binary liquids and metal alloys. The rate of change of a bubble's volume is proportional to its surface area and to its Laplace pressure difference with respect to a certain mean or critical bubble size: $\mathrm{d} R^{d} / \mathrm{d} t \propto R^{d-1}\left(1 / R_{c}-1 / R\right)$, or $\mathrm{d} R / \mathrm{d} t \propto\left(1 / R_{c}-1 / R\right)$, for any dimension $d$; thus large bubbles $R>R_{c}$ grow while small bubbles $R<R_{c}$ shrink. Note that this is a "mean-field" theory, in which each bubble gets its marching orders by comparison with an average rather than by comparison with its actual neighbors. The next step is to combine this rate of change with an expression of total gas conservation to arrive at a differential equation for the evolution of the distribution of bubble sizes. The result predicts that an initially-narrow distribution will smoothly broaden until reaching a stationary state, in which the shape of the distribution is constant and the average bubble radius grows as the square-root of time. In particular, note that this approach does not predict a bidisperse regime at intermediate times.

In the stationary polydisperse regime, the evolution of the average bubble radius may be modeled as $\mathrm{d} R_{a} / \mathrm{d} t=D_{o} F(\varepsilon) / R_{a}$. Here $D_{o}$ is a materials constant with units of $\mathrm{cm}^{2} / \mathrm{s}$; it is proportional to the gas diffusivity and solubility, and is inversely proportional to film 


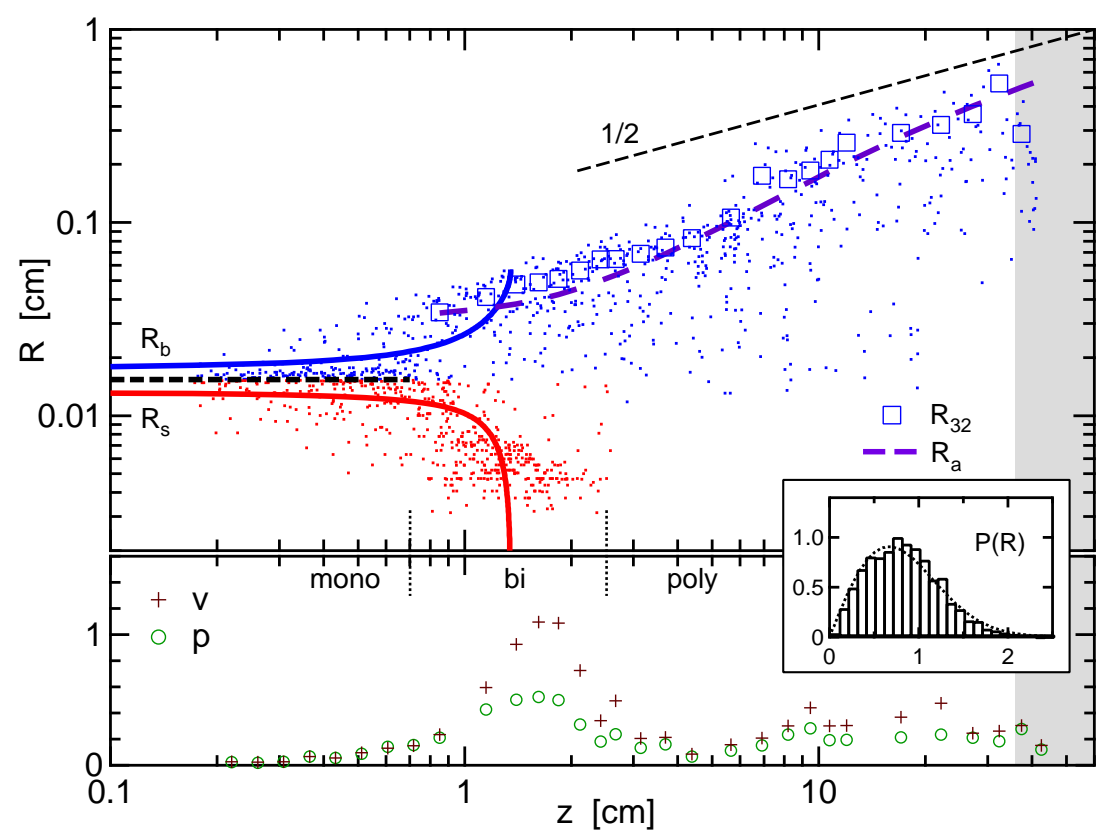

Fig. 3 - TOP: Scatter plot of individual bubble radii vs height; ratios of third to second moments of the distribution, $R_{32}$, are shown by open squares. The average radius at the very bottom is $\langle R\rangle=0.0154 \mathrm{~cm}$, as given by the horizontal dashed black line. The standard deviation at the very bottom is $\sigma_{o}=0.0022 \mathrm{~cm}$. The solid blue and red curves, which represent solution of Eqs. (24), approach $\langle R\rangle \pm \sigma_{o}$ at $z=0$. The dashed purple curve represents solution of Eq. (1). The gray area represents the top of the sample, where bubbles burst. BOTTOM: Height dependence of the polydispersity parameters $v=\left\langle R^{2}\right\rangle /\langle R\rangle^{2}-1$ and $p=\left\langle R^{3}\right\rangle^{2 / 3} /\left\langle R^{2}\right\rangle-1$. INSET: Distribution of bubble radii in the polydisperse region; the $\mathrm{x}$-axis is scaled by $R_{32}$, which is related to the average radius by $R_{32} /\langle R\rangle=1.38 \pm 0.13$. The dashed curve is a fit to a Weibull distribution, $\propto R \exp \left(-k R^{2}\right)$.

thickness. Three different forms have been suggested for the liquid-fraction dependence of the coarsening rate: $F(\varepsilon)=1-\sqrt{\varepsilon / 0.36}[4], F(\varepsilon)=(1-\sqrt{\varepsilon / 0.44})^{2}[5]$, and $F(\varepsilon)=1 / \sqrt{\varepsilon}[6]$. The former two are based on total blockage of diffusion by the Plateau borders; the latter is based on scaling of pressure differences with the average Plateau border curvature. Direct measurements of the coarsening rate [6] support $F(\varepsilon)=1 / \sqrt{\varepsilon}$ but do not definitively rule out the other two forms. However, our new scatter data for bubble radius vs height contradict these with no analysis whatsoever. If the coarsening rate were constant for very dry foams, as these suggest, then we'd expect $\mathrm{d} R_{a} / \mathrm{d} t \propto 1 / R_{a}$; this implies $R_{a}(t) \sim t^{1 / 2}$ and hence $R_{a}(z) \sim z^{1 / 2}$ in our experiment. Since the bubble radii data in Fig. 3 grow faster than $z^{1 / 2}$, the coarsening rate must continue to increase for ever-drier foams.

Altogether our observations in the polydisperse regime are thus best modeled by

$$
R_{a} \mathrm{~d} R_{a} / \mathrm{d} t=D_{o} / \sqrt{\varepsilon}
$$

where $D_{o}$ is the only unknown parameter. It has been suggested that the most suitable average radius is given by the ratio of third to second moments of the bubble radius distribution, $R_{32}=\left\langle R^{3}\right\rangle /\left\langle R^{2}\right\rangle$, known as the Sauter mean radius [19]. We compute this at each height for which the liquid fraction was measured. Then we directly compute $D_{o}$ from Eq. (11) as the 
height-average of $\sqrt{\varepsilon} R_{32}\left(u \mathrm{~d} R_{32} / \mathrm{d} z\right)$. The result is $D_{o}=(3 \pm 1) \times 10^{-6} \mathrm{~cm}^{2} / \mathrm{s}$. For illustration, this value was used with Eq. (1) to generate the dashed purple curve in Fig. [3 giving the growth of the Sauter radius starting from the observed value at the smallest height measured. This prediction grows faster than $z^{1 / 2}$ and agrees quite well with $R_{32}$ data throughout the column.

Now we turn attention to the mono- and bidisperse regimes near the bottom of the column. The simplest model we can conceive that goes beyond mean field is one in which there are precisely two sizes of bubbles, such that each big bubble $R_{b}$ grow at the sole expense of $N$ neighboring small bubbles $R_{s}$. For a tractable model, we imagine a one-dimensional situation in which gas conservation is expressed as

$$
N R_{s}+R_{b}=(N+1) R_{o}
$$

and where the right-hand side remains constant as $R_{s}$ shrinks and $R_{b}$ grows. To complete the model, we take the time evolution of the small bubbles according to the Laplace pressure difference and the above liquid-fraction dependence as

$$
\frac{\mathrm{d} R_{s}}{\mathrm{~d} t}=\frac{\alpha D_{o}}{\sqrt{\varepsilon}}\left(\frac{1}{R_{b}}-\frac{1}{R_{s}}\right) .
$$

Here $\alpha$ is a dimensionless parameter whose value is expected to be of order 1. Substituting $R_{b}$ from Eq. (2), separating variables, and integrating with initial condition $R_{s}(0)=R_{o}-\delta_{o}$, gives an exact solution for the evolution of $R_{s}(t)$ :

$$
\frac{\alpha D_{o} t}{\sqrt{\varepsilon}}=\frac{R_{o}{ }^{2}}{N+1}\left\{\frac{\left(R_{o}-\delta_{o}-R_{s}\right)\left[N\left(R_{o}-\delta_{o}+R_{s}\right)-2 R_{o}\right]}{2 R_{o}{ }^{2}}+\ln \left(\frac{R_{o}-R_{s}}{\delta_{o}}\right)\right\} .
$$

If $R_{s}$ is treated as the independent variable, then time $t$ and height $z=u t$ can be generated from Eq. (4) while $R_{b}$ can be generated from Eq. (2).

We compare our model with data as follows, adjusting as few parameters as possible. The initial conditions are taken from the average $\langle R\rangle=0.0154 \mathrm{~cm}$ and standard deviation $\sigma_{o}=0.0022 \mathrm{~cm}$ of the bubble sizes at the very bottom of the column, such that $R_{s}(0)=$ $\langle R\rangle-\sigma_{o}=0.0132 \mathrm{~cm}$ and $R_{b}(0)=\langle R\rangle+\sigma_{o}=0.0176 \mathrm{~cm}$. In light of gas conservation Eq. (2), this corresponds to $R_{o}=\langle R\rangle-\sigma_{o}(N-1) /(N+1)$ and $\delta_{o}=2 \sigma_{o} /(N+1)$. The value of $N$ is then deduced from a parametric plot of big vs small bubble radii at equal heights in the bidisperse range. The form of $R_{b}$ vs $R_{s}$ is linear, and best fit gives $N=3.0 \pm 0.5$. Altogether the parameters in the prediction Eq. (44) are thus fixed as $D_{o}=3 \times 10^{-6} \mathrm{~cm}^{2} / \mathrm{s}$, $\mathrm{N}=3, R_{o}=0.0143 \mathrm{~cm}$, and $\delta_{o}=0.0011 \mathrm{~cm}$. The only unknown adjustable parameter is $\alpha$. The best fit gives $\alpha=0.4 \pm 0.1$, as illustrated by the solid red and blue curves in Fig. [3 The agreement with the scatter data is quite satisfactory across the mono- and bidisperse regimes.

Conclusions. - In summary we observe the bubble size distribution in a steady-state column of foam to evolve from monodisperse, to bidisperse, to polydisperse, as a function of height. The bidisperse region was unexpected, but, once formed, its evolution may be understood in term of the evaporation of small bubbles into neighboring large bubbles. In addition we observe the rate of coarsening in the polydisperse regime to increase with decreasing liquid fraction, without detected bound, consistent with $R_{a} \mathrm{~d} R_{a} / \mathrm{d} t=D_{o} / \sqrt{\varepsilon}$. These findings expand the current understanding of coarsening and shed light onto its interplay with drainage phenomena. Furthermore they underscore the importance of the bubble size distribution, and the nontrivial role it plays. Our findings also raise new questions. How does a bidisperse foam emerge from a nearly monodisperse initial condition? It appears that the fastest growing "mode" is one in which essentially alternate bubbles shrink and grow in a spatially-correlated 
manner. Is this generic, or is it due to extraordinary conspiracy of drainage and coarsening in steady-state foams? Could it be due to segregation of less-soluble impurities in the gas [20]? Could it be due to bidisperse pressures for equal-volume bubbles, as in the Weaire-Phelan A15 foam [21,22]? This latter effect seems most likely to us, but suitably generalized to random structures. Because of Plateau's rules all the films cannot have a mean curvature of zero. For every bubble face which bows out there is an inward face on the adjacent bubble. It follows that there are two distributions of faces with negative and positive curvature, respectively. Thus in a dry foam with equal volume bubbles, there must be a distribution of bubble curvatures and hence pressures. This scenario emphasizes the lack of connection between bubble size and pressure. Resolving these issues would have fundamental interest, and could aid applications in which steady-state foams must be controlled for efficient fractionation of chemicals and particulates.

We thank A. Saint-Jalmes for helpful conversations. This work was supported by NASA (NNC04GB61G, KF and DJD), NSF (DMR05-47230, OLH and RDK), and the ACS Petroleum Research Fund (OLH and RDK).

\section{REFERENCES}

[1] Stavans, J., Rep. Prog. Phys., 56 (1993) 733.

[2] Weaire, D. and Hutzler, S., The Physics of Foams (Clarendon Press, Oxford) 1999.

[3] Saint-Jalmes, A., Vera, M. U., and Durian, D. J., Europhys. Lett., 50 (2000) 695.

[4] Hutzler, S. and Weaire, D., Phil. Mag. Lett., 80 (2000) 419.

[5] Hilgenfeldt, S., Koehler, S. A. and Stone, H. A., Phys. Rev. Lett., 86 (2001) 4704.

[6] Vera M. U. and Durian D. J., Phys. Rev. Lett., 88 (2002) 088304.

[7] Pilon L., Fedorov, A. G. and Viskanta, R., J. Coll. I. Sci., 242 (2001) 425.

[8] Barbian, N., Ventura-Medina, E., and Cilliers, J. J., Min. Eng., 16 (2003) 1111.

[9] Grassia, P., Neethling, S. J., Cervantes, C. and Lee, H. T., Coll. Surf. A - Physicochem. Eng. Asp., 274 (2006) 110.

[10] Feitosa, K., Marze, S., Saint-Jalmes, A. and Durian, D. J., J. Phys. Cond. Mat., 17 (2005) 6301.

[11] Cheng, H. C. and Lemlich, R., Ind. Eng. Chem. Fundam., 22 (1983) 105.

[12] Glazier, J.A., Anderson, M. P., and Grest, G. S., Phil. Mag. B, 62 (1990) 615.

[13] Magrabi, S. A., Dlugogorski, B. Z. and Jameson, G. J., Chem. Eng. Sci., 54 (1999) 4007.

[14] Ganan-Calvo, A. M., Fernandez, J. M., Oliver, A. M. and Marquez, M., Appl. Phys. Lett., 84 (2004) 4989.

[15] Glazier, J. A., Gross, S. P. and Stavans, J., Phys. Rev. A, 36 (1987) 306.

[16] Stavans, J. and Glazier, J. A., Phys. Rev. Lett., 62 (1989) 1318.

[17] Lemlich, R., Ind. Eng. Chem. Fundam., 17 (1978) 89.

[18] Markworth, A. M., J. Coll. I. Sci., 107 (1985) 569.

[19] Kraynik, A. M., Reinelt, D. A. and van Swol, F., Phys. Rev. Lett., 93 (2004) 208301.

[20] Weaire, D. and Pageron, V., Phil. Mag. Lett., 62 (1990) 417.

[21] Weaire, D. and Phelan, R., Phil. Mag. Lett., 69 (1994) 107.

[22] Levine, D. and Grest, G. S., Phil. Mag. Lett., 74 (1996) 303. 